\title{
Obovatol inhibits the growth and aggressiveness of tongue squamous cell carcinoma through regulation of the EGF-mediated JAK-STAT signaling pathway
}

\author{
MINGLI DUAN $^{1}$, XIAOMING DU ${ }^{2}$, GANG REN $^{1}$, YONGDONG ZHANG ${ }^{1}$, \\ YU ZHENG ${ }^{1}$, SHUPING $\mathrm{SUN}^{2}$ and JUN ZHANG ${ }^{2}$ \\ ${ }^{1}$ Department of Stomatology, Tianjin First Center Hospital Dental, Tianjin, Hebei 300192; \\ ${ }^{2}$ Department of Maxillofacial Surgery, Tianjin Stomatological Hospital and Maxillofacial Surgery, \\ Tianjin, Hebei 300041, P.R. China
}

Received November 25, 2016; Accepted December 18, 2017

DOI: $10.3892 / \mathrm{mmr} .2018 .9078$

\begin{abstract}
Migration and invasion are the most important characteristics of human malignancies which limit cancer drug therapies in the clinic. Tongue squamous cell carcinoma (TSCC) is one of the rarest types of cancer, although it is characterized by a higher incidence, rapid growth and greater potential for metastasis compared with other oral neoplasms worldwide. Studies have demonstrated that the phenolic compound obovatol exhibits anti-tumor effects. However, the potential mechanisms underlying obovatol-mediated signaling pathways have not been completely elucidated in TSCC. The present study investigated the anti-tumor effects and potential molecular mechanisms mediated by obovatol in TSCC cells and tissues. The results of the present study demonstrated that obovatol exerted cytotoxicity in SCC9 TSCC cells, and inhibited their migration and invasion. In addition, obovatol induced apoptosis in SCC9 TSCC cells by increasing caspase 9/3 and apoptotic protease enhancing factor 1 expression levels. Western blot analysis demonstrated that obovatol inhibited the expression of pro-epidermal growth factor (EGF), Janus kinase (JAK), and signal transducer and activator of transcription (STAT) in SCC9 TSCC cells. A study of the molecular mechanisms demonstrated that depletion of EGF reversed the obovatol-mediated inhibition of SCC9 TSCC cell growth and aggressiveness. Animal experiments indicated that obovatol significantly inhibited TSCC tumor growth and increased the number of apoptotic cells in tumor tissues. In conclusion, the
\end{abstract}

Correspondence to: Professor Xiaoming Du, Department of Maxillofacial Surgery, Tianjin Stomatological Hospital and Maxillofacial Surgery, 73 Heiniucheng Road, Hexi, Tianjin, Hebei 300041, P.R. China

E-mail: duxiaomingtianjin@yeah.net

Key words: tongue squamous cell carcinoma, obovatol, apoptosis, pro-epidermal growth factor, Janus kinase-signal transducer and activator of transcription results of the present study provided scientific evidence that obovatol inhibited TSCC cell growth and aggressiveness through the EGF-mediated JAK-STAT signaling pathway, suggesting that obovatol may be a potential anti-TSCC agent.

\section{Introduction}

Oral cancer is characterized by high incidence and mortality rates in developing countries, and is the 11th most common cancer in the world (1). Tongue squamous cell carcinoma (TSCC) is the most common oral malignancy with different histopathological symptoms and etiopathogenesis of tumorigenesis $(2,3)$. Epidemiological data have indicated alterations in the demographic profile of patients suffering from TSCC (4). Although numerous advanced anticancer treatments have been proposed for use in the clinic, TSCC has been traditionally considered to be associated with frequent recurrence and metastasis, and a poor prognosis, due to rapid migration and invasion (5). Local migration to parenchymal tissues and long distance metastasis to organs are the principal reasons underlying the mortality rate of TSCC and the poor survival rate among patients with TSCC (6). Therefore, understanding the potential mechanisms of TSCC cell growth and aggressiveness is important to inhibit tumor metastasis and improve survival for patients.

Obovatol is a phenolic compound extracted from the bark of Magnolia obovata, which is renowned for its pharmacodynamic functions of antioxidation, neuroprotection, antithrombosis, anti-inflammation and antitumor. Previously, obovatol has been observed to exhibit growth inhibitory effects on tumor cells and tissues through the induction of apoptotic cell death $(7,8)$. A study demonstrated that obovatol inhibited prostate and colon cancer cell growth by inducing apoptosis and inhibition of the nuclear factor- $\mathrm{\kappa B}$ signaling pathway (9). Another report indicated that obovatol inhibited colorectal cancer growth and aggressiveness by suppressing tumor cell proliferation and inducing apoptosis, and that it may therefore be a potent inducer of tumor cell apoptosis and a potent antitumor agent (10). Additionally, obovatol may induce apoptosis in non-small cell lung cancer cells via C/EBP homologous 
protein activation, activated caspase 9/3 and apoptosis regulator Bax, and that it attenuated the expression of cyclin D1 in A549 and H460 non-small cell lung cancer cells (11).

The present study investigated the inhibitory effects of obovatol on the growth and aggressiveness of SCC9 TSCC cells . The present study examined obovatol-mediated pro-epidermal growth factor (EGF)-mediated Janus kinase (JAK)-signal transducer and activator of transcription (STAT) signaling in SCC9 TSCC cells. The results demonstrated that obovatol may induce apoptosis in SCC9 TSCC cells by increasing caspase 9/3 and apoptotic protease enhancing factor 1 (Apaf-1) expression levels mediated by the EGF-induced JAK-STAT signaling pathway.

\section{Materials and methods}

Ethics statement. The present preclinical study was performed according to the recommendations in the Guide for the Tianjin First Center Hospital (Dental; Tianjin, China). All experimental protocols and animals were approved by the Committee on the Ethics of Animal Experiments in Defence Research of Tianjin First Center Hospital (TFCH-023/0016; Tianjin, China). All surgery was performed under intravenous sodium pentobarbital $(37 \mathrm{mg} / \mathrm{kg})$. The present study was additionally approved by the Ethical Committee of Tianjin Stomatological Hospital and Maxillofacial Surgery (Tianjin, China).

Cells and reagents. SCC9 cells were purchased from the American Type Culture Collection (Manassas, VA, USA) and tumor cells were cultured in minimum essential medium (MEM; Sigma-Aldrich; Merck KGaA, Darmstadt, Germany) supplemented with $10 \%$ fetal calf serum (Invitrogen; Thermo Fisher Scientific, Inc., Waltham, MA, USA). Cells were cultured in an incubator at $37^{\circ} \mathrm{C}$ and $5 \% \mathrm{CO}_{2}$.

MTT assay. SCC9 cells (6x10 3 cells) were treated with obovatol $(5.00 \mathrm{mg} / \mathrm{ml})$ in 96 -well plates for $24 \mathrm{~h}$ in triplicate for each condition, with PBS as a control. MTT (20 $\mu \mathrm{l} ; 5 \mathrm{mg} / \mathrm{ml})$ was added to each well subsequent to incubation at $37^{\circ} \mathrm{C}$. All plates were further incubated for $4 \mathrm{~h}$ and the medium was subsequently removed, and $100 \mu \mathrm{l}$ dimethyl sulfoxide was added into the wells to solubilize the crystals. The optical density was measured using a ELISA reader (Bio-Rad Laboratories, Inc., Hercules, CA, USA) at wavelength of $450 \mathrm{~nm}$.

Reverse transcription-quantitative polymerase chain reaction (RT-qPCR). Total mRNA was isolated from SCC9 cells using an RNA Easy Mini Extract kit (Sigma-Aldrich; Merck KGaA). RNA samples with a A260/A280 ratio between 1.8 and 2.0 were used to synthesize cDNA via RT using SuperScript III Reverse Transcriptase (Thermo Fisher Scientific, Inc.). Reaction conditions for RT were: $65^{\circ} \mathrm{C}$ for $5 \mathrm{~min}, 50^{\circ} \mathrm{C}$ for $35 \mathrm{~min}$ and $85^{\circ} \mathrm{C}$ for $5 \mathrm{~min}$. The expression of fibronectin (FN), vimentin (VM), E-cadherin (ED), EGF and JAK in SCC9 cells was detected using an SYBR ${ }^{\circledR}$ Green Real-Time PCR Master Mixes (Thermo Fisher Scientific, Inc.), with $\beta$-actin expression as an endogenous control. All procedures were performed according to the manufacturer's protocol. qPCR reaction conditions were: $95^{\circ} \mathrm{C}$ for $45 \mathrm{sec}$ (initial denaturation), followed by 40 cycles of $95^{\circ} \mathrm{C}$ for $15 \mathrm{sec}$ (denaturation), $60^{\circ} \mathrm{C}$ for $45 \mathrm{sec}$ (anealing/elongation) and $72^{\circ} \mathrm{C}$ for $30 \mathrm{sec}$ (final extension). All the primers were synthesized by Invitrogen (Invitrogen; Thermo Fisher Scientific, Inc.). Primer sequences were: FN forward, 5'-CCCACCGTCTCA ACATGCTTA G-3', reverse 5'-CTCGGCTTCCTCCATAACAAGTAC-3'; VM forward, 5'-TCTACGAGGAGGAGATGCGG-3', reverse 5'-GGTCAAGACGTGCCAGAGAC-3'; ED forward, 5'-GTC AGTTCAGACTCCAGCCC-3', reverse 5'-AAATTCACTCTG CCCAGGACG-3'; EFG forward, 5'-GTGCAGCTTCAGGAC CACAA-3', reverse 5'-AAATGCATGTGTCGAATATCTTGA G-3'; JAK forward, 5'-AAGCTTTCTCACAAGCATTTGGTT T-3', reverse 5'-AGAAAGGCATTAGAAAGCCTGTAGTT-3' and $\beta$-actin forward, 5'-GACCTCTATGCCAACACAGT-3' and reverse 5'-AGTACTTGCGCTCAGGAGGA-3. Relative mRNA expression levels were determined using $2^{-\Delta \Delta C q}(12)$. The final results were presented in the $\mathrm{n}$-fold manner compared with $\beta$-actin.

Cells invasion and migration assays. SCC9 cells were incubated with obovatol $(5.00 \mathrm{mg} / \mathrm{ml})$. SCC9 cells were suspended at a density of $1 \times 10^{6}$ in $500 \mu \mathrm{l}$ serum-free MEM. Cells were seeded at the top of BD BioCoat Matrigel Migration Chambers (BD Biosciences, Franklin Lakes, NJ, USA), and MEM containing 20\% fetal calf serum (Sigma-Aldrich, Merck $\mathrm{KGaA}$ ) was applied to the lower chamber. Cells were incubated at $37^{\circ} \mathrm{C}$ for $24 \mathrm{~h}$ and $0.5 \%$ crystal violet (Sigma-Aldrich; Merck $\mathrm{KGaA}$ ) staining was performed at room temperature for $20 \mathrm{~min}$ to analyze cell migration, according to the manufacturer's protocol. For the migration assay, SCC9 cells were seeded into a control insert (BD Biosciences) instead of a Matrigel Migration Chamber. SCC9 cell migration and invasion was counted in at least three randomly stained fields using a light microscope (magnification, x100) (Olympus Corporation, Tokyo, Japan) for every membrane.

Small interfering RNA (siRNA) transfections. SCC9 cells ( $5 \times 10^{5}$ cells in $100 \mu 1 \mathrm{MEM}$ ) were cultured to $85 \%$ confluence and transfected with siRNA (40 nM; 5'-CAGCATCTG TCTAATCAAA-3') targeting EGF (EGFKD) or scrambled siRNA (5'-AATCAATCCATCCTT-3') by incubating with Lipofectamine $^{\mathrm{TM}}$ RNAi MAX (Invitrogen; Thermo Fisher Scientific, Inc.) at $37^{\circ} \mathrm{C}$ in a $\mathrm{CO}_{2}$ incubator for $4 \mathrm{~h}$ according to the manufacturer's protocols, followed by incubation in MEM at $37^{\circ} \mathrm{C}$ for $48 \mathrm{~h}$ prior to harvest. siRNA targeting EGF and scrambled siRNA were obtained from GenePharma (Shanghai, China).

Western blotting. SCC9 cells were homogenized in radioimmunoprecipitation assay buffer (Thermo Fisher Scientific, Inc.) containing protease inhibitor and were centrifuged at $3,800 \mathrm{x} \mathrm{g}$ at $4^{\circ} \mathrm{C}$ for $10 \mathrm{~min}$. The supernatant of the mixture was used for the analysis of protein expression. Concentration of protein samples was measured by Bicinchoninic Acid assay. Then, 10\% SDS-PAGE gel electrophoresis was performed using $30 \mu \mathrm{g}$ protein from each sample, followed by transfer to a polyvinylidene difluoride membrane. For western blotting, primary antibodies (Abcam, Cambridge, UK) were added following blocking with $5 \%$ skimmed milk for $1 \mathrm{~h}$ at $37^{\circ} \mathrm{C}$. Primary antibodies included: Rabbit anti-CDK1 (1:1,000; ab131450, Abcam), anti-CDK2 (1:1,000; ab64669, Abcam), 
anti-FN (1:1,000; ab23750, Abcam), anti-VM (1:1,000; ab137321, Abcam), anti-ED (1:1,000; ab1416, Abcam), anti-Caspase-3 (1:1,000; ab13847, Abcam), anti-Caspase-9 (1:1,000; ab2324, Abcam), anti-Apaf-1 (1:1,000; ab53152, Abcam), anti-p53 (1:1,000; ab131442, Abcam), anti-Bcl-2 (1:1,000; ab32124, Abcam), anti-JAK (1:1,000; ab125051, Abcam), anti-STAT (1:1,000; ab32520, Abcam), anti-phosphorylated (p)JAK (1:1,000; ab138005, Abcam), anti-pSTAT (1:1,000; ab76315, Abcam), anti-PDGF (1:1,000; ab124392, Abcam), anti-VEGFR (1:1,000; ab32152, Abcam), anti-EFG (1:1,000; ab10409, Abcam), and anti- $\beta$ actin $(1: 1,000$; ab6276, Abcam). The membrane was incubated with all mentioned primary antibodies for overnight $(8-10 \mathrm{~h})$ at $37^{\circ} \mathrm{C}$. Membranes were subsequently incubated with horseradish peroxidase-conjugated anti-immunoglobulin $\mathrm{G}$ for $24 \mathrm{~h}$ at $4^{\circ} \mathrm{C}$ (1:10,000; ab6721, Abcam). The results were visualized using a chemiluminescence detection system (LumiGLO; Cell Signaling Technology, Inc., Danvers, MA, USA). Relative expression levels of each protein were normalized to endogenous control $\beta$-actin using ImageJ v2 (National Institutes of Health, Bethesda, MD, USA).

Apoptosis assay. SCC9 cells were grown to $90 \%$ confluence. Apoptosis was assessed by incubating the cells with obovatol $(5.00 \mathrm{mg} / \mathrm{ml})$ for $24 \mathrm{~h}$. Following incubation, SCC9 cells were trypsinized and collected. The cells were washed in PBS, adjusted to $1 \times 10^{6}$ cells $/ \mathrm{ml}$ and labeled with annexin V-fluorescein isothiocyanate (FITC) and propidium iodide (annexin V-FITC kit; BD Biosciences). SCC9 cells were analyzed using a FACScan flow cytometer (BD Biosciences). The percentage of labeled SCC9 cells undergoing apoptosis in each group was determined and calculated using BD Cell Quest pro software v5.1 (BD Biosciences).

Animal study. Male Balb/c (specific pathogen free) nude mice (age, 6-8 weeks, 20-25 g) were purchased from Shanghai SLAC Laboratory Animal Co., Ltd. (Shanghai, China). Mice were raised in laminar airflow cabinets under a $12 \mathrm{~h}$ light/dark cycle at a constant temperature of $20-26^{\circ} \mathrm{C}$ and humidity of $30-70 \%$. Animals were fed an ad libitum diet of irradiated and sterilized dry food and sterile drinking water. SCC9 cells $\left(1 \times 10^{7}\right)$ were mixed with $100 \mu 1$ PBS and injected subcutaneously into the flanks of Balb/c mice $(n=80)$. Xenografted mice were divided into two groups ( $\mathrm{n}=40 \mathrm{mice} / \mathrm{group}$ ) and received treatment with obovatol $(5.00 \mathrm{mg} / \mathrm{kg})$ once a day for 6 days following tumor implantation (diameter, $5-8 \mathrm{~mm}$ ). The tumor volumes were calculated according to a previous method (13). A fraction of the mice ( $\mathrm{n}=10$ mice/group) were sacrificed and tumor sections were obtained for further analysis on day 24. The remaining animals ( $\mathrm{n}=30 \mathrm{mice} / \mathrm{group}$ ) were housed until day 120 to observe the survival rate of experimental mice. Control mice were not treated.

Immunohistochemistry. TSCC tissues isolated from experimental mice were fixed in formaldehyde (10\%) for $24 \mathrm{~h}$ at room temperature, followed by embedding in paraffin. Tumor tissues were sliced into tumor sections with a thickness of $8 \mu \mathrm{m}$. Antigen retrieval was performed by incubating with $10 \mathrm{mM}$ citrate buffer ( $\mathrm{pH} 6.0$ ) at $95-100^{\circ} \mathrm{C}$ for $10 \mathrm{~min}$. Slides were allowed to cool for $20 \mathrm{~min}$, followed by two washes with
PBS, 5 min each and xylene was used to deparaffinize the tissue, prior to rehydration by descending series of alcohol. Then tissue sections were blocked using $10 \%$ fetal bovine serum (Invitrogen; Thermo Fisher Scientific, Inc.) in PBS at room temperature for $1 \mathrm{~h}$, followed by incubation with primary antibodies, including rabbit anti-Caspase-3, anti-Caspase-9, anti-Apaf-1, anti-EFG, anti-JAK and anti-STAT. Subsequently, tissues were washed with PBS twice for 5 min per wash and incubated with secondary antibody to Rabbit Immunoglobulin G-H\&L (Biotin, 1:1,000, ab97049, Abcam) in a humidified chamber at room temperature for $30 \mathrm{~min}$. Following washing, color development was performed by adding 3,3'-Diaminobenzidine (DAB; Sigma-Aldrich; Merck KGaA). Signals were observed under a light microscope (magnification, 20x, Olympus). A Ventana Benchmark automated staining system (Ventana Medical Systems, Inc., Tucson, AZ, USA) was used for observation of protein expression levels.

Terminal deoxynucleotidyl transferase dUTP nick end labeling (TUNEL) to detect renal cell apoptosis. Paraffin-embedded sections (10\% formaldehyde-fixed) were subjected to antigen retrieval following dewaxing and dehydration as aforementioned. The tissue sections were incubated with proteinase $\mathrm{K}(20 \mu \mathrm{g} / \mathrm{ml}$ in Tris/HCl, $\mathrm{pH}$ 7.4-8.0 from Thermo Fisher Scientific, Inc.) at room temperature for 15-30 min and washed twice with PBS. Subsequently, the tissues were placed in methanol solution containing $3 \% \mathrm{H}_{2} \mathrm{O}_{2}$ at room temperature for $5 \mathrm{~min}$, followed by washing with PBS for $1 \mathrm{~min}$. The TUNEL reaction mixture $(50 \mu \mathrm{l})$ was added and incubated at $37^{\circ} \mathrm{C}$ with tissue in a wet box for $60 \mathrm{~min}$, followed by 3 washes with PBS. Transformation pod/TUNEL pod (50 $\mu 1$; Sigma-Aldrich; Merck KGaA) was then added and incubated at $37^{\circ} \mathrm{C}$ with tissue in a wet box for $60 \mathrm{~min}$, followed by 3 washes with PBS. Finally, DAB substrate solution (50-100 $\mu \mathrm{l})$ was added and incubated with tissue at room temperature for 10 min, followed by 3 washes with PBS. Hematoxylin staining was performed at $37^{\circ} \mathrm{C}$ for $15 \mathrm{~min}$ and the slides were sealed. The sealed slides were observed under microscope.

Statistical analysis. All data are presented as the mean \pm standard deviation of triplicate dependent experiments, and were analyzed using Student's t-tests or one-way analysis of variance (Tukey honest significant difference post hoc test). All data were analyzed using SPSS 19.0 software (IBM Corp., Armonk, NY, USA) and GraphPad Prism version 5.0 (GraphPad Software, Inc., La Jolla, CA, USA), in addition to Microsoft Excel 2010 (Microsoft Corporation, Redmond, WA, USA). $\mathrm{P}<0.05$ was considered to indicate a statistically significant difference.

\section{Results}

Obovatol inhibits TSCC cell growth and aggressiveness through cell cycle arrest and downregulation of metastasis-associated protein expression. The inhibitory effects of obovatol on the growth and aggressiveness of TSCC cells were analyzed in the present study. The results demonstrated that obovatol significantly inhibited SCC9 TSCC cell growth (Fig. 1A). The expression levels of cyclin dependent kinase (CDK)1 and CDK2 were downregulated in SCC9 TSCC cells 

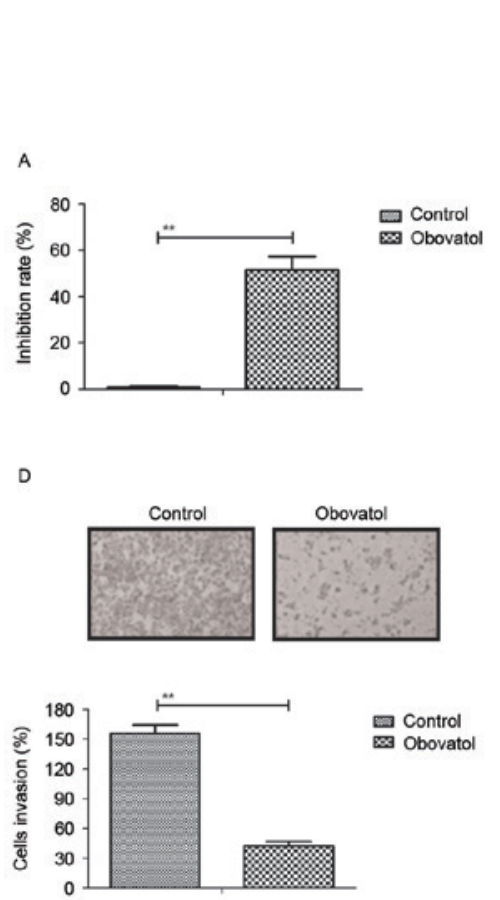
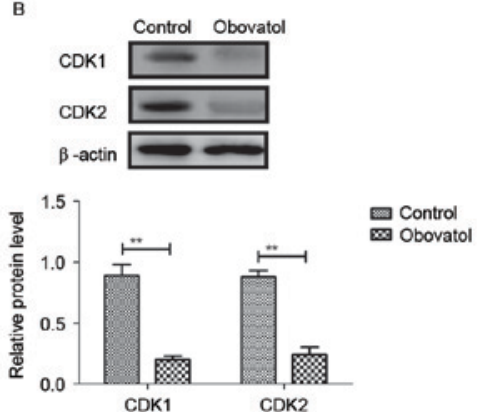

(1)
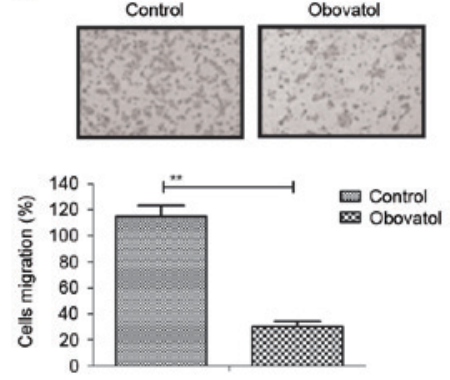

$\mathbf{F}$

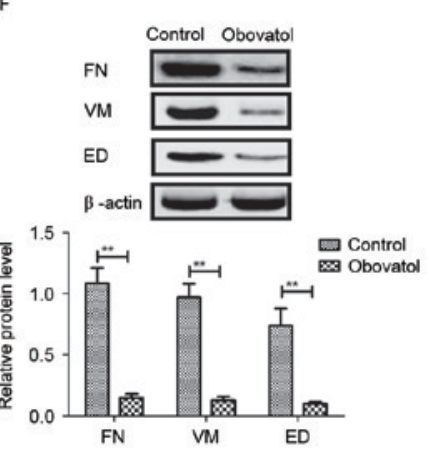

Figure 1. Effects of obovatol on TSCC cell growth and aggressiveness. (A) Effects of obovatol on SCC9 TSCC cell growth. (B) Effects of obovatol on the expression levels of CDK1 and CDK2 in SCC9 TSCC cells. The effects of obovatol on (C) migration (magnification, x20) and (D) invasion (magnification, $\mathrm{x} 20$ ) of SCC9 TSCC cells were investigated. The (E) mRNA and (F) protein expression levels of FN, VM and ED in obovatol-treated SCC9 TSCC cells were determined. All data are presented as the mean \pm standard error of the mean of triplicate samples. ${ }^{* *} \mathrm{P}<0.01$ : Control vs. Obovatol. TSCC, tongue squamous cell carcinoma; CDK, cyclin-dependent kinase; FN, fibronectin; VM, vimentin; ED, E-cadherin.

following obovatol administration (Fig. 1B). As presented in Fig. 1C and D, migration and invasion assays demonstrated that treatment with obovatol suppressed the migration and invasion of SCC9 TSCC cells. It was additionally observed that the expression levels of FN, VM and ED were decreased by treatment with obovatol, as determined by RT-qPCR and western blot analyses (Fig. 1E-F). The results of the present study indicated that obovatol administration inhibited the growth and aggressiveness of SCC9 TSCC cells.

Obovatol induces apoptosis in TSCC cells through regulation of apoptosis-associated gene expression. The pro-apoptotic effects of obovatol in TSCC cells were analyzed in the present study. The results demonstrated that treatment with obovatol promoted apoptosis in SCC9 TSCC cells (Fig. 2A). Cell viability analysis demonstrated that obovatol enhanced the cellular atrophy of SCC9 TSCC cells (Fig. 2B). It was observed that caspase-3, caspase-9 and Apaf-1 expression levels were increased in SCC9 TSCC cells, as determined by western blotting (Fig. 2C). The results additionally demonstrated that the expression levels of p53 and $\mathrm{Bcl}-2$ were downregulated following obovatol administration in SCC9 TSCC cells (Fig. 2D). These investigations suggested that treatment with obovatol promoted apoptosis in SCC9 TSCC cells by increasing pro-apoptotic gene expression and decreasing anti-apoptotic gene expression.

Obovatol inhibits the expression of EGF and downregulates the JAK-STAT signaling pathway in TSCC cells. Previous studies suggested that EGF expression levels, and JAK-STAT transcriptional activation and mRNA stabilization, may be associated with the proliferation and metastasis of human carcinoma cells (14-16). Therefore, the present study analyzed EGF expression and the JAK-STAT signaling pathway in SCC9 TSCC cells. As presented in Fig. 3A and B, the mRNA and protein levels of EGF were decreased by treatment with obovatol in SCC9 TSCC cells. The results additionally demonstrated that the mRNA and protein expression levels of JAK and STAT were downregulated in obovatol-treated SCC9 TSCC cells (Fig. 3C and D). Treatment with obovatol decreased the phosphorylation levels of JAK and STAT in SCC9 TSCC cells (Fig. 3E). It was additionally observed that platelet-derived growth factor (PDGF) and vascular endothelial growth factor receptor (VEGFR) protein expression levels were downregulated by treatment with obovatol (Fig. 3F). These data suggested that obovatol may inhibit the expression of EGF and downregulate the JAK-STAT signaling pathway in TSCC cells.

Obovatol inhibits TSCC cellular apoptosis through the EGF-mediated JAK-STAT signaling pathway. In order to identify the mechanism through which the EGF-mediated JAK-STAT signaling pathway may be inhibited by obovatol, alterations in the expression and phosphorylation levels of JAK and STAT were analyzed in EGF-knockdown SCC9 TSCC cells. As presented in Fig. 4A and B, EGF knockdown (EGFKD) inhibited the obovatol-mediated decreased expression and phosphorylation levels of JAK and STAT in SCC9 TSCC cells. Expression levels of caspase-3, caspase-9 and Apaf-1 were increased in the EGFKD group compared with the EGFKD-OB group (Fig. 4C). Notably, the obovatol-induced increase in apoptosis was abolished in 
A
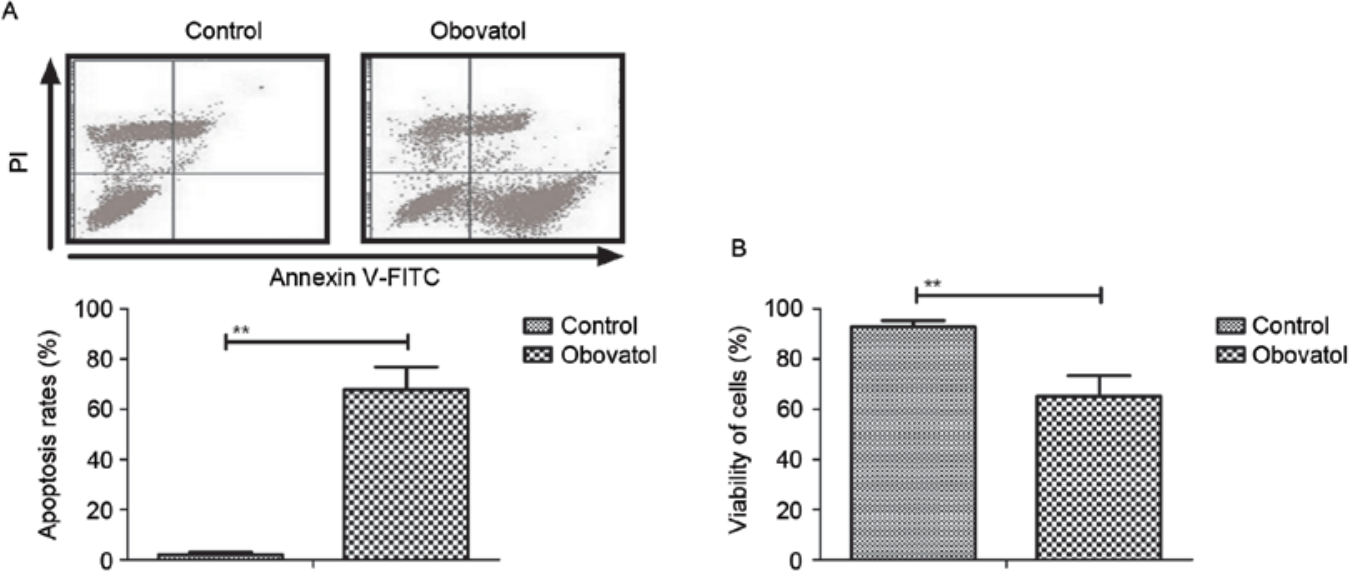

C

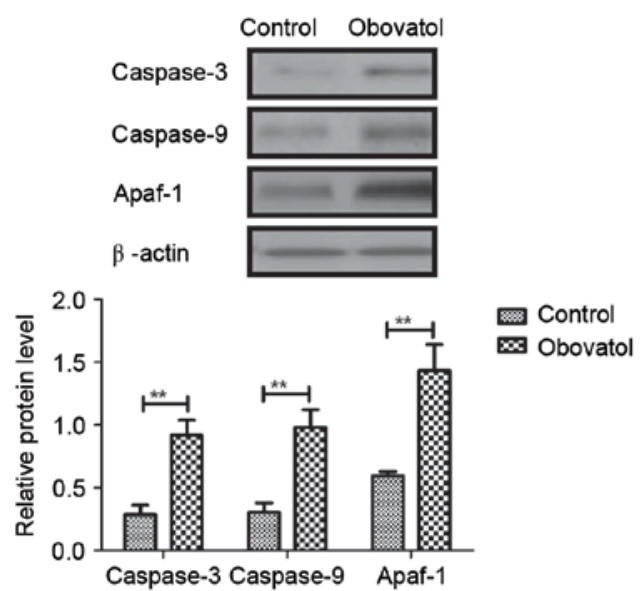

D

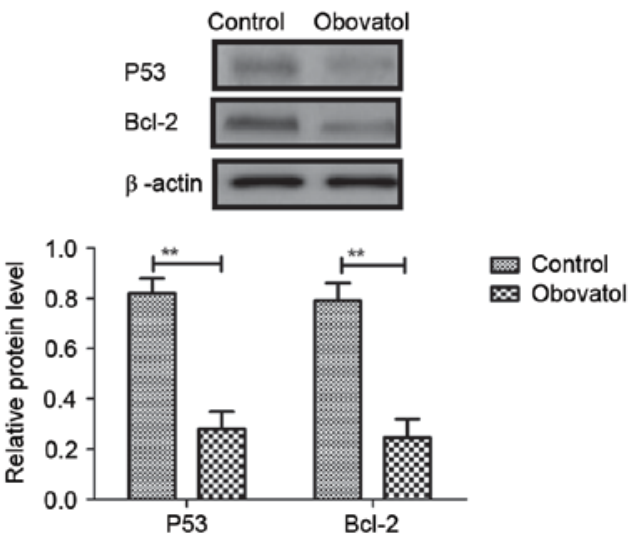

Figure 2. Obovatol induces apoptosis in SCC9 TSCC cells through regulation of apoptosis-associated gene expression. (A) Treatment with obovatol promotes apoptosis in SCC9 TSCC cells. (B) Effects of obovatol on the viability of SCC9 TSCC cells. (C) Effects of obovatol on caspase-3, caspase-9 and Apaf-1 expression levels in SCC9 TSCC cells. (D) Effects of obovatol on the expression levels of p53 and Bcl-2 in SCC9 TSCC cells. All data are presented as the mean \pm standard error of the mean of triplicate samples. ${ }^{* *} \mathrm{P}<0.01$ : Control vs. Obovatol. TSCC, tongue squamous cell carcinoma; PI, propidium iodide; FITC, fluorescein isothiocyanate; Apaf-1, apoptotic protease enhancing factor 1; p53, cellular tumor antigen p53; Bcl-2, apoptosis regulator Bcl-2.

EGF-knockdown SCC9 TSCC cells (Fig. 4D). These results indicated that treatment with obovatol induced TSCC cellular apoptosis through the EGF-mediated JAK-STAT signaling pathway.

In vivo anti-cancer effects of obovatol in xenografted mice. The anti-tumor efficacy of treatment with obovatol was studied in SCC9-bearing mice. The results demonstrated that treatment with obovatol $(12 \mathrm{mg} / \mathrm{kg})$ significantly inhibited tumor growth in a 24-day experiment (Fig. 5A). Treatment with obovatol promoted cellular apoptosis in tumors, as determined by TUNEL assay (Fig. 5B). Immunohistochemistry demonstrated that caspase-3, caspase- 9 and Apaf-1 expression levels were upregulated in obovatol-treated tumor tissues compared with controls (Fig. 5C). The results demonstrated that treatment with obovatol decreased the expression levels of EGF, JAK and STAT in tumor tissues (Fig. 5D). The results additionally demonstrated that PDGF and VEGFR protein expression levels were downregulated by treatment with obovatol in tumor tissues (Fig. 5E). Notably, it was observed that treatment with obovatol prolonged the survival of tumor-bearing mice in a 120-day observation (Fig. 5F). These outcomes suggested that obovatol may exhibit in vivo anti-cancer effects and prolong survival in tumor-bearing mice.

\section{Discussion}

Epidemiology has indicated that the morbidity and mortality rate of TSCC is increasing in young people (16). The current management and treatment strategy for the majority of patients with TSCC is partial surgical glossectomy, followed by radiotherapy and chemotherapy in the clinic (17-19). However, the rapid growth of TSCC cells, local migration towards adjacent tissues and long-distance metastasis to the neck, lymphatic system and other organs, shortens the five-year survival period $(20,21)$. A previous study indicated that inhibiting a number of signaling pathways in TSCC cells may be regarded as a potential biomarker and therapeutic target in mobile TSCC (22). Evidence has demonstrated that obovatol may be regarded as an anti-tumor agent, with the potential to inhibit tumor cell growth by inducing apoptosis and arresting the cell cycle in tumor cells (9). In the present study, the inhibitory effects of treatment with obovatol in TSCC cells and tissues were analyzed in vitro and in vivo. The present study 

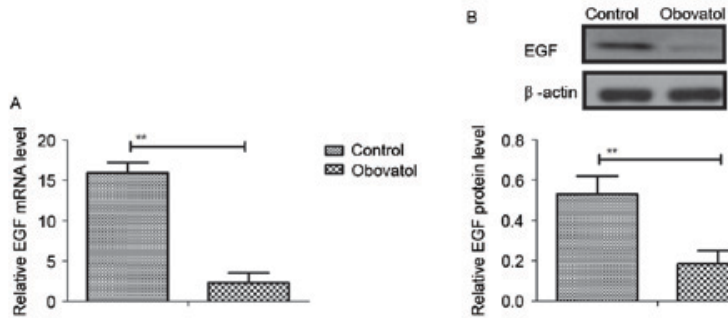

Control
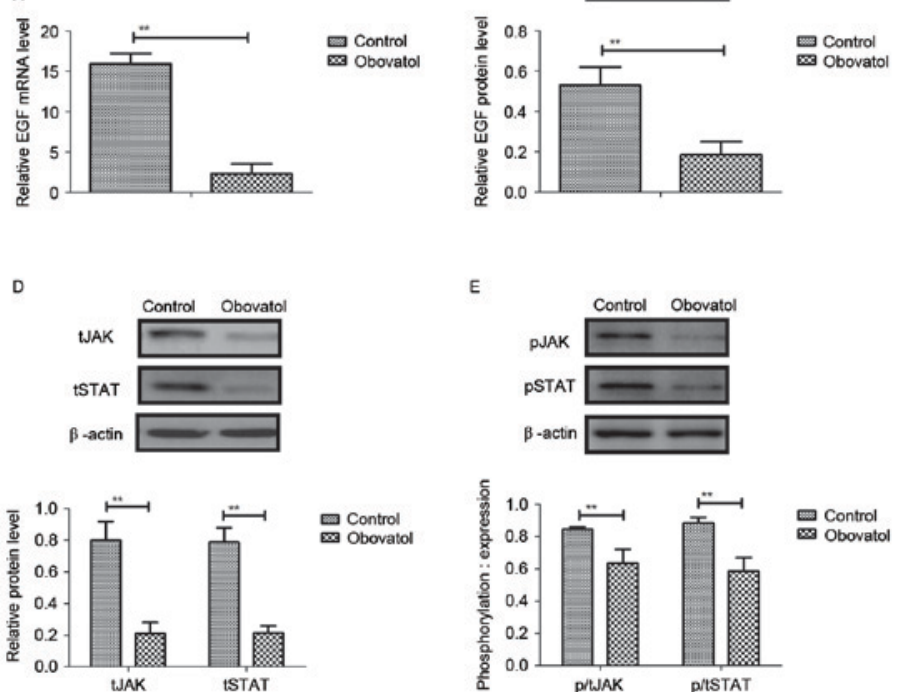

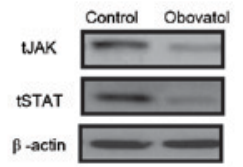

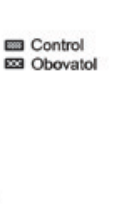

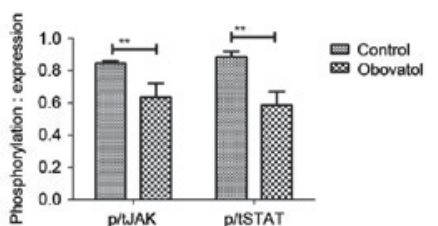

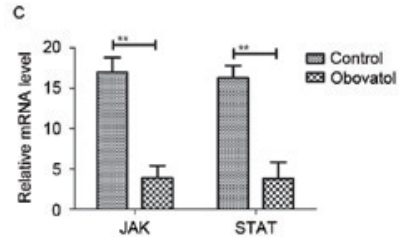

$F$
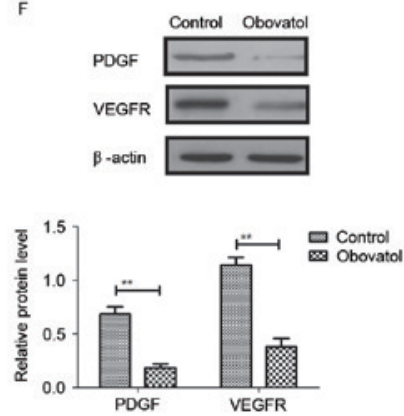

Figure 3. Effects of obovatol on the expression levels of EGF, JAK and STAT in SCC9 TSCC cells. The effects of obovatol on the (A) mRNA and (B) protein expression levels of EGF in SCC9 TSCC cells were investigated. The effects of obovatol on the (C) mRNA and (D) protein expression levels of JAK and STAT in SCC9 TSCC cells. (E) Treatment with obovatol treatment decreased the phosphorylation levels of JAK and STAT in SCC9 TSCC cells. (F) Treatment with obovatol downregulated the expression levels of PDGF and VEGFR in SCC9 TSCC cells. All data are presented as the mean \pm standard error of the mean of triplicate samples. ${ }^{* *} \mathrm{P}<0.01$ : Control vs. Obovatol. TSCC, tongue squamous cell carcinoma; EGF, pro-epidermal growth factor; JAK, Janus kinase; STAT, signal transducer and activator of transcription; PDGF, platelet-derived growth factor; VEGFR, vascular endothelial growth factor receptor; p, phosphorylated.

A
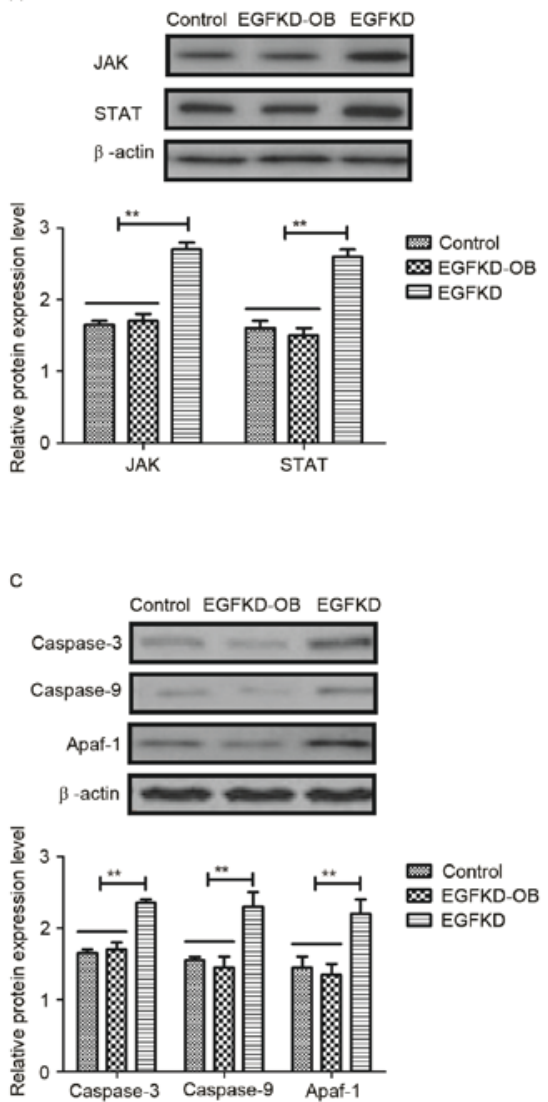
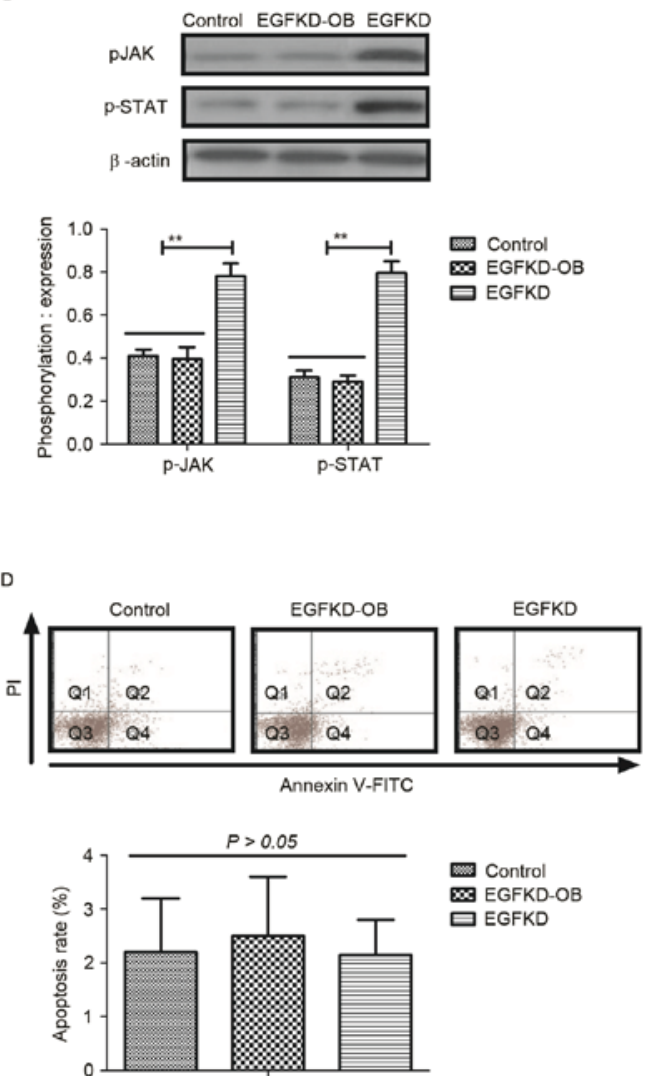

Figure 4. Obovatol regulates SCC9 TSCC cellular apoptosis through the EGF-mediated JAK-STAT signaling pathway. The effects of EGF knockdown on the (A) expression and (B) phosphorylation levels of JAK and STAT in SCC9 TSCC cells. (C) The expression levels of caspase-3, caspase-9 and Apaf-1 in EGF-knockdown SCC9 TSCC cells. (D) Obovatol-induced apoptosis was inhibited by knockdown of EGF in SCC9 TSCC cells. All data are presented as the mean \pm standard error of the mean of triplicate samples. ${ }^{* *} \mathrm{P}<0.01$ : EGDKD vs. EGDKD-OB. TSCC, tongue squamous cell carcinoma; EGF, pro-epidermal growth factor; JAK, Janus kinase; STAT, signal transducer and activator of transcription; Apaf-1, apoptotic protease enhancing factor 1; KD, knockdown; FITC, fluorescein isothiocyanate; PI, propidium iodide; p, phosphorylated; OB, treated with obovatol. 

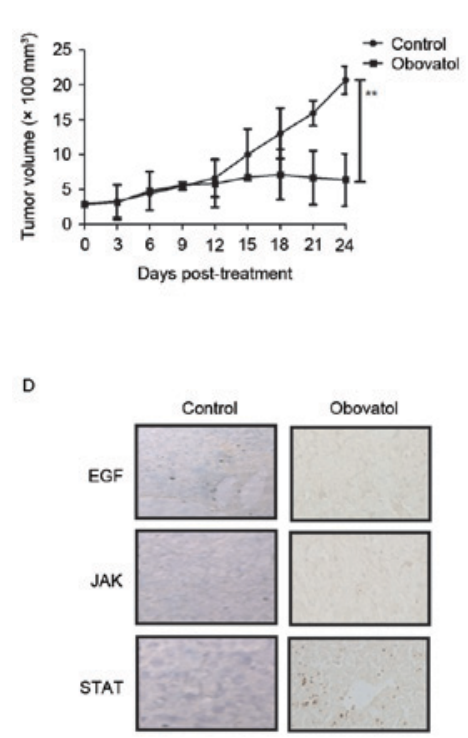
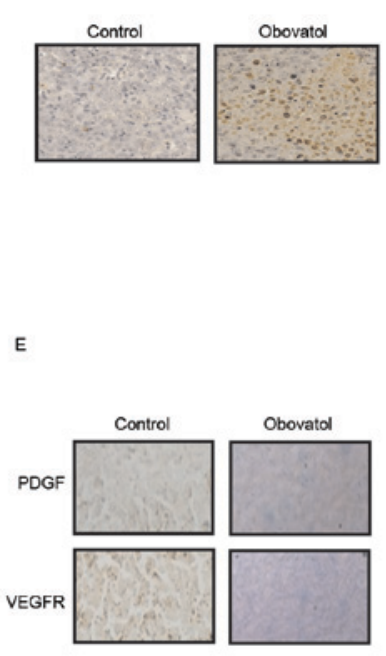
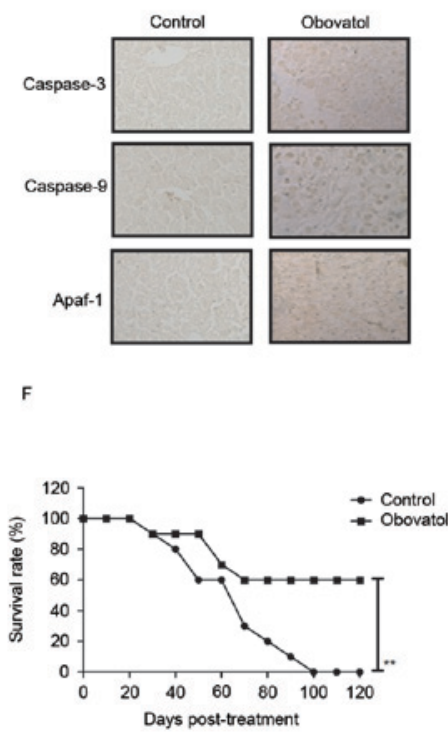

Figure 5. In vivo anti-cancer efficacy of treatment with obovatol in TSCC cell-bearing mice. (A) Treatment with obovatol inhibited tumor growth in 24-day experiments. (B) Treatment with obovatol promoted cellular apoptosis in tumor tissues (magnification, x20). (C) Immunohistochemistry was used to analyze the effects of obovatol on caspase-3, caspase-9 and Apaf-1 expression levels in tumors tissues (magnification, x20). (D) Effects of treatment with obovatol on EGF, JAK and STAT in tumor tissues (magnification, x20). (E) Effects of treatment with obovatol on PDGF and VEGFR protein expression levels in tumor tissues (magnification, x20). (F) Treatment with obovatol prolonged the survival of SCC9-bearing mice in a 120-day experiment. $\mathrm{n}=8$ animals/group. All data are presented as the mean \pm standard error. ${ }^{* *} \mathrm{P}<0.01$ : Control vs. Obovatol. TSCC, tongue squamous cell carcinoma; EGF, pro-epidermal growth factor; JAK, Janus kinase; STAT, signal transducer and activator of transcription; Apaf-1, apoptotic protease enhancing factor 1; PDGF, platelet-derived growth factor; VEGFR, vascular endothelial growth factor receptor.

additionally investigated the potential mechanism underlying obovatol-mediated apoptosis stimulation and growth inhibition in SCC9 TSCC cells. The results indicated that treatment with obovatol inhibited growth and aggressiveness, and additionally promoted apoptosis in TSCC cells through the EGF-mediated JAK-STAT signaling pathway. In vivo assays demonstrated that treatment with obovatol significantly suppressed tumor growth and prolonged survival, suggesting that obovatol may be a promising anti-cancer agent for TSCC therapy.

At present, reports have indicated that obovatol may efficiently inhibit the growth of various human cancer cells through regulation of cellular signaling pathways $(10,11)$. Lee et al $(23)$ reported that obovatol enhanced docetaxel-induced prostate and colon cancer cell death through inactivation of nuclear transcription factor $-\kappa \mathrm{B}$, and suggested that obovatol may possess therapeutic potential in combination with other antineoplastic chemotherapeutics. Arora et al (24) indicated that the role of obovatol in arresting the cell cycle may potentiate the cytotoxic effects and induce apoptosis in human pancreatic cancer cells. An additional study demonstrated that treatment with obovatol inhibited cell proliferation and induced cell death by decreasing the phosphorylation of RAC- $\alpha$ serine/threonine-protein kinase and serine/threonine-protein kinase mTOR, which further inhibited $\gamma$-secretase activity by downregulating the expression of $\gamma$-secretase complex proteins, particularly $\gamma$-secretase subunit aph-1, in malignant melanoma cancer cells (24). Although these reports have presented the inhibitory effects of obovatol in human cancer, the underlying anti-tumor mechanism is not well understood in TSCC cells.

Studies have demonstrated that the inhibition of EGF expression contributes to the inhibition of tumor growth and further inhibits tumor metastasis $(25,26)$. Al-Hazzaa et al (27) reported that EGF receptor tyrosine kinase inhibitor ZD1839 promoted cisplatin-induced apoptosis in SCC-15 cells. In addition, it has been reported that targeting of the JAK-STAT signaling pathway may induce tumor cell apoptosis and inhibit tumor cell proliferation in tumor bearing mice treated with mBIIB036 (28). Furthermore, Hernandez et al (29) demonstrated that tumor growth and aggressiveness was able to be mediated through JAK-STAT-interferon signaling pathways in HCT116 cells. In the present study, the results indicated that obovatol downregulated p53 and Bcl-2 expression levels in TSCC cells, which led to increased apoptosis in SCC9 TSCC cells $(30,31)$. It was additionally observed that obovatol suppressed EGF expression, and the expression and phosphorylation levels of JAK and STAT in SCC9 TSCC cells. Obovatol treatment abolished the upregulation of caspase-3, caspase-9 and Apaf-1 expression levels in SCC9 TSCCs induced by EG knockdown. The present study design identified that treatment with obovatol induced TSCC cellular apoptosis through the EGF-mediated JAK-STAT signaling pathway.

In conclusion, the present study indicated that treatment with obovatol efficiently inhibited TSCC cell growth through downregulation of the expression levels of CDK1 and CDK2. The aggressiveness of TSCC cells was suppressed by obovatol administration via decreased expression of FN, VM and ED. The findings additionally indicated that obovatol administration induced apoptosis in TSCC cells through inhibition of the EGF-mediated JAK-STAT signaling pathway, which further contributed to the inhibition of tumor growth in vivo and prolonged the survival of tumor-bearing mice. Overall, the results of the present study suggested that 
obovatol administration may be a potential anti-cancer agent for the treatment of TSCC via blockade of the EGF-mediated JAK-STAT signal pathway.

\section{Acknowledgements}

Not applicable.

\section{Funding}

No funding was received.

\section{Availability of data and materials}

The datasets used and/or analyzed during the current study are available from the corresponding author on reasonable request.

\section{Authors' contributions}

MD, JZ and XD made substantial contributions to the concept and design of the present study. GR, MD and YDZ made substantial contributions to the conception of the present study and conducted data analysis. YZ, SS and JZ performed statistical analysis. Also, XD and JZ drafted the manuscript. All authors read and approved the final manuscript.

\section{Ethics approval and consent to participate}

The present study was approved by the Committee on the Ethics of Animal Experiments in Defence Research of Tianjin First Center Hospital (TFCH-023/0016).

\section{Consent for publication}

Not applicable.

\section{Competing interests}

The authors declare that they have no competing interests.

\section{References}

1. Dik EA, Willems SM, Ipenburg NA, Rosenberg AJ, Van Cann EM and van Es RJ: Watchful waiting of the neck in early stage oral cancer is unfavourable for patients with occult nodal disease. Int J Oral Maxillofac Surg 45: 945-950, 2016.

2. Chen G, Cai X, Ren JG, Jia J and Zhao YF: Unexpected development of tongue squamous cell carcinoma after sclerotherapy for the venous malformation: A unique case report and literature review. Diagn Pathol 8: 182, 2013.

3. Kradin RL, Sheldon TA, Nielsen P, Selig M and Hunt J: Malacoplakia of the tongue complicating the site of irradiation for squamous cell carcinoma with review of the literature. Ann Diagn Pathol 16: 214-218, 2012.

4. Murphy J, Berman DR, Edwards SP, Prisciandaro J, Eisbruch A and Ward BB: Squamous cell carcinoma of the tongue during pregnancy: A case report and review of the literature. J Oral Maxillofac Surg 74: 2557-2566, 2016.

5. Martínez C, Hernández M, Martínez B and Adorno D: Frequency of oral squamous cell carcinoma and oral epithelial dysplasia in oral and oropharyngeal mucosa in Chile. Rev Med Chil 144 169-174, 2016 (In Spanish).

6. Shrestha A, Marla V, Shrestha S and Agrawal D: Awareness of undergraduate dental and medical students towards oral cancer. J Cancer Educ 32: 778-783, 2017.
7. Lim Y, Kwon JS, Kim DW, Lee SH, Park RK, Lee JJ, Hong JT, Yoo HS, Kwon BM and Yun YP: Obovatol from Magnolia obovata inhibits vascular smooth muscle cell proliferation and intimal hyperplasia by inducing p21Cip1. Atherosclerosis 210: 372-380, 2010.

8. Choi DY, Lee YJ, Lee SY, Lee YM, Lee HH, Choi IS, Oh KW, Han SB, Nam SY and Hong JT: Attenuation of scopolamine-induced cognitive dysfunction by obovatol. Arch Pharm Res 35: 1279-1286, 2012.

9. Lee SY, Yuk DY, Song HS, Yoon DY, Jung JK, Moon DC, Lee BS and Hong JT: Growth inhibitory effects of obovatol through induction of apoptotic cell death in prostate and colon cancer by blocking of NF-kappaB. Eur J Pharmacol 582: 17-25, 2008.

10. Lee SK, Kim HN, Kang YR, Lee CW, Kim HM, Han DC, Shin J, Bae K and Kwon BM: Obovatol inhibits colorectal cancer growth by inhibiting tumor cell proliferation and inducing apoptosis. Bioorg Med Chem 16: 8397-8402, 2008.

11. Kim H, Shin EA, Kim CG, Lee DY, Kim B, Baek NI and Kim SH: Obovatol induces apoptosis in non-small cell lung cancer cells via C/EBP homologous protein activation. Phytother Res 30: 1841-1847, 2016.

12. Livak KJ and Schmittgen TD: Analysis of relative gene expression data using real-time quantitative PCR and the 2(-Delta Delta C(T)) method. Methods 25: 402-408, 2001.

13. Bai FL, Yu YH, Tian H, Ren GP, Wang H, Zhou B, Han XH, Yu QZ and Li DS: Genetically engineered Newcastle disease virus expressing interleukin-2 and TNF-related apoptosis-inducing ligand for cancer therapy. Cancer Biol Ther 15: 1226-1238, 2014.

14. Xiao YS, Zhou J, Fan J, Sun QM, Zhao Y, Sun RX, Liu YK and Tang ZY: Interferon-alpha upregulates thymidine phosphorylase expression via JAK-STAT transcriptional activation and mRNA stabilization in human hepatocellular carcinoma SMMC-7721 cells. Zhonghua Zhong Liu Za Zhi 30: 444-447, 2008 (In Chinese).

15. Saxena NK, Sharma D, Ding X, Lin S, Marra F, Merlin D and Anania FA: Concomitant activation of the JAK/STAT, $\mathrm{PI} 3 \mathrm{~K} / \mathrm{AKT}$, and ERK signaling is involved in leptin-mediated promotion of invasion and migration of hepatocellular carcinoma cells. Cancer Res 67: 2497-2507, 2007.

16. Kalinowski FC, Giles KM, Candy PA, Ali A, Ganda C, Epis MR, Webster RJ and Leedman PJ: Regulation of epidermal growth factor receptor signaling and erlotinib sensitivity in head and neck cancer cells by miR-7. PloS one 7: e47067, 2012.

17. $\mathrm{Ng} \mathrm{JH}$, Iyer NG, Tan MH and Edgren G: Changing epidemiology of oral squamous cell carcinoma of the tongue: A global study. Head Neck 39: 297-304, 2017.

18. Wong WM, Parvathaneni U, Jewell PD, Martins RG, Futran ND, Laramore GE and Liao JJ: Squamous cell carcinoma of the oral tongue in a patient with Fanconi anemia treated with radiotherapy and concurrent cetuximab: A case report and review of the literature. Head Neck 35: E292-E298, 2013.

19. Iyengar NM, Ghossein RA, Morris LG, Zhou XK, Kochhar A, Morris PG, Pfister DG, Patel SG, Boyle JO, Hudis CA and Dannenberg AJ: White adipose tissue inflammation and cancer-specific survival in patients with squamous cell carcinoma of the oral tongue. Cancer 122: 3794-3802, 2016.

20. Goepfert RP, Kezirian EJ and Wang SJ: Oral tongue squamous cell carcinoma in young women: A matched comparison-do outcomes justify treatment intensity? ISRN Otolaryngology 2014: 529395, 2014

21. Seppälä M, Pohjola K, Laranne J, Rautiainen M, Huhtala H, Renkonen R, Lemström K, Paavonen T and Toppila-Salmi S: High relative density of lymphatic vessels predicts poor survival in tongue squamous cell carcinoma. Eur Arch Otorhinolaryngol 273: 4515-4524, 2016.

22. Theocharis S, Giaginis C, Dana E, Thymara I, Rodriguez J, Patsouris E and Klijanienko J: Phosphorylated epidermal growth factor receptor expression is associated with clinicopathologic parameters and patient survival in mobile tongue squamous cell carcinoma. J Oral Maxillofac Surg 75: 632-640, 2017.

23. Lee SY, Cho JS, Yuk DY, Moon DC, Jung JK, Yoo HS, Lee YM, Han SB, Oh KW and Hong JT: Obovatol enhances docetaxel-induced prostate and colon cancer cell death through inactivation of nuclear transcription factor-kappaB. J Pharmacol Sci 111: 124-136, 2009.

24. Arora S, Bhardwaj A, Srivastava SK, Singh S, McClellan S, Wang B and Singh AP: Honokiol arrests cell cycle, induces apoptosis, and potentiates the cytotoxic effect of gemcitabine in human pancreatic cancer cells. PloS one 6: e21573, 2011. 
25. Gotz R and Sendtner M: Cooperation of tyrosine kinase receptor TrkB and epidermal growth factor receptor signaling enhances migration and dispersal of lung tumor cells. PloS One 9: e100944, 2014.

26. Andersson N, Anttonen M, Färkkilä A, Pihlajoki M, Bützow R, Unkila-Kallio L and Heikinheimo M: Sensitivity of human granulosa cell tumor cells to epidermal growth factor receptor inhibition. J Mol Endocrinol 52: 223-234, 2014.

27. Al-Hazzaa A, Bowen ID, Randerson P and Birchall MA: The effect of ZD1839 (Iressa), an epidermal growth factor receptor tyrosine kinase inhibitor, in combination with cisplatin, on apoptosis in SCC-15 cells. Cell Prolif 38: 77-86, 2005.

28. Chapman MS, Wu L, Amatucci A, Ho SN and Michaelson JS: TWEAK signals through JAK-STAT to induce tumor cell apoptosis. Cytokine 61: 210-217, 2013.
29. Hernandez JM, Elahi A, Clark W, Humphries LA, Wang J, Achille A, Seto E and Shibata D: The tumor suppressive effects of HPP1 are mediated through JAK-STAT-interferon signaling pathways. DNA Cell Biol 34: 541-549, 2015.

30. Fourati A, El May MV, Ben Abdallah M, Gamoudi A, Mokni N, Goucha A, Boussen H, Ladgham A and El May A: Prognostic evaluation of $\mathrm{p} 53$, heat shock protein $70, \mathrm{Ki} 67$, and CD34 expression in cancer of the tongue in Tunisia. J Otolaryngol Head Neck Surg 38: 191-196, 2009.

31. Lin YT, Yang JS, Lin HJ, Tan TW, Tang NY, Chaing JH, Chang YH, Lu HF and Chung JG: Baicalein induces apoptosis in SCC-4 human tongue cancer cells via a Ca2+-dependent mitochondrial pathway. In Vivo 21: 1053-1058, 2007. 